\title{
Molecular characteristic and expression analysis of collagenolytic serine protease from the Chinese mitten crab Eriocheir sinensis with defense response to Vibrio anguillarum challenge
}

\author{
Q.Z. Yang ${ }^{1,2}$, Z.J. Yang', Y. Zhang ${ }^{1}$, X.L. Li ${ }^{3}$ and W. Zhang ${ }^{1}$ \\ ${ }^{1}$ School of Basic Medical Science, Xinxiang Medical University, \\ Xinxiang, Henan, China \\ ${ }^{2}$ Sanquan College of Xinxiang Medical University, Xinxiang, Henan, China \\ ${ }^{3}$ Xinxiang Vocational and Technical College, Xinxiang, Henan, China \\ Corresponding author: W. Zhang \\ E-mail: wzhang.cn@hotmail.com
}

Genet. Mol. Res. 13 (2): 3885-3894 (2014)

Received June 4, 2013

Accepted October 1, 2013

Published April 29, 2014

DOI http://dx.doi.org/10.4238/2014.April.29.1

\begin{abstract}
A novel collagenolytic serine protease (CLSP) was cloned from the hemocytes of the Chinese mitten crab Eriocheir sinensis (EsCLSP). The full-length cDNA of Es-CLSP contains 990 nucleotides. It encodes a 270-amino acid-long peptide with the mature peptide containing 221 amino acids. It contains the conserved catalytic triad (H, D, and S). Similarity analysis shows that Es-CLSP shares high identity with CLSPs from the fiddler crab Uca pugilator. Es-CLSP mRNA expression in E. sinensis is a) tissue-related with the highest expression in hemocytes and widely distributed, b) highly responsive to Vibrio anguillarum challenge in hemocytes, and c) a different response to the intruding pathogens. The results of this study demonstrate the successful isolation of Es-CLSP and indicate that Es-CLSP is an immune-related gene, and show the possible role of CLSP in the invertebrate innate immune system.
\end{abstract}

Key words: Collagenolytic serine protease; Eriocheir sinensis; Innate immunity 


\section{INTRODUCTION}

Invertebrate phenoloxidase exists in blood in an inactive form, which is activated in a stepwise process involving serine proteinases (SPs) (Söderhäll and Cerenius, 1998). SP constitutes one of the largest families of enzymes in the animal kingdom (Barrett and Rawlings, 1995; Hedstrom, 2001). They play important roles in immune response (Finnerty et al., 1999; O'Connell et al., 2006), food protein digestion (Broehan et al., 2007), embryogenesis (Sveinsdóttir et al., 2006), and other biological progress in insects (Herrero et al., 2005). SPs are characterized by an active site called the catalytic triad, which contains H, D, and S amino acids (Sellos and Van Wormhoudt, 1999). The serine residue at the active site participates in the formation of a transient acylenzyme intermediate between the substrate and protease (Botos et al., 2000). Collagenolytic serine protease (CLSP) is a kind of SPs. In mammals, it participates in immune reactions and many other physiological functions (Barrett and Rawlings, 1995). Studies on CLSPs in invertebrates have mainly focused on insects; this is because many insects are agricultural pests and studying their digestive systems may aid develop methods for crop protection (Herrero et al., 2005). The CLSP from Manduca sexta also participates in the degradation of Cry toxin, a type of insecticidal crystal protein produced by Bacillus thuringiensis (Miranda et al., 2001). In Drosophila, a CLSP is involved in immune defense reactions against bacteria (de Morais et al., 2005; Moffitt et al., 2012). Recently, more and more CLSPs have been discovered in invertebrates (Sellos and Van Wormhoudt, 1992; Colebatch et al., 2002; Zhu et al., 2005; Broehan et al., 2007; Shi et al., 2008; Danwattananusorn et al., 2009; Yadav et al., 2011). CLSPs have been cloned in the shrimp and their polymorphism patterns and immune response to bacteria have been analyzed (Sellos and Van Wormhoudt, 1992; Van Wormhoudt et al., 1992; Shi et al., 2008). However, little is known about the roles of this protease in immune defense in the Chinese mitten crab.

The Chinese mitten crab Eriocheir sinensis is native to China. This crab is a traditional savory food especially in the Yangtze River Area, and is one of the most economically important freshwater aquatic species of China. With the development of intensive E. sinensis culture, which has rapidly expanded over the last 2 decades, the yield has reached a value of $4.0 \times 10^{5} \mathrm{t}$ in China in 2005. However, various diseases caused by bacteria (especially Gramnegative bacteria) or rickettsia-like organisms have also begun to emerge and have resulted in enormous losses (Chen et al., 2007). Therefore, an improved understanding of the innate immune ability of crabs and their bio-defense mechanisms has become a research priority (García-Ulloa et al., 2003). The worldwide crab culture industry has been beset with diseases mainly caused by bacteria, especially Vibrio anguillarum, and has suffered significant economic losses. In recent years, many cases of natural infection of Chinese mitten crab by $V$. anguillarum have been recorded in Zhejiang, Jiangsu, and other areas of China (Chen et al., 2007). Improving immunity and disease resistance in crustaceans is one of the fundamental measures for prevention and control of diseases. Although studies on the epidemiology and prevention of bacterial infections have been performed (Sánchez-Paz, 2010), a detailed analysis of molecular resistance mechanism to V. anguillarum in E. sinensis has not been reported. In this study, we cloned a CLSP from E. sinensis, and determined its transcript expression patterns to ascertain the role of Es-CLSP in its innate immune defense mechanism against $V$. anguillarum. 


\section{MATERIAL AND METHODS}

\section{Tissue collection and nucleic acid preparation}

Healthy adult Chinese mitten crabs were collected from the Xinxiang aquatic product market from September to October 2012 in Xinxiang, China. After acclimatization for 1 week at $20-25^{\circ} \mathrm{C}$ in aerated freshwater, the crabs were placed in an ice bath for 3-5 min until each crab was lightly anesthetized. Hemolymph was drawn from the hemocoel in the arthrodial membrane of the last pair of walking legs by using a syringe (approximately $2.0 \mathrm{~mL}$ per crab) with an equal volume of anticoagulant solution $(2.15 \mathrm{~g}$ glucose, $0.8 \mathrm{~g}$ citrate, $0.43 \mathrm{~g} \mathrm{NaCl}$, $100 \mathrm{~mL}$ double-distilled water) added, and centrifuged at $800 \mathrm{~g}$ at $4^{\circ} \mathrm{C}$ to isolate hemocytes. The other tissues (the hepatopancreas, gills, testis, accessory gland, ovary, muscle, stomach, intestine, heart, thoracic ganglia, and brain) were harvested, snap frozen in liquid nitrogen, and stored at $-80^{\circ} \mathrm{C}$ prior to nucleic acid analysis. For cloning and expression analyses, tissue samples from 5 crabs were pooled and ground with a mortar and pestle prior to extraction.

For bacterial challenge, 120 crabs were divided equally into 2 groups: the experimental group crabs were injected in the arthrodial membrane of the last pair of walking legs with approximately $100 \mathrm{~mL}(500 \mathrm{mg} / \mathrm{mL}$, L2630, Sigma-Aldrich) suspended in phosphate-buffered saline (PBS), while control group was injected with $100 \mathrm{~mL}$ PBS $(\mathrm{pH}=7.4)$. Five crabs were randomly selected at each time interval of 0 (as blank control), 2, 4, 8, 12, 24, 48, and $72 \mathrm{~h}$ post-injection. The hepatopancreas was harvested according to the above-described method and stored at $-80^{\circ} \mathrm{C}$. Excluding the 40 crabs sacrificed for tissue collection, the experimental group had 9 deaths, and the control group had 0 death until $72 \mathrm{~h}$ after the challenge. Total RNA was extracted from E. sinensis by using Trizol reagent (RNA Extraction kit, Invitrogen, USA) according to manufacturer's protocol. For cloning and expression analyses, the organs from 5 crabs were pooled and ground with a mortar and pestle prior to RNA extraction. Total RNA (2 $\mu \mathrm{g})$ isolated from various organs were reversely transcribed using the Prime Script ${ }^{\mathrm{TM}}$ RT-PCR kit or the Prime Script Real-time quantitative PCR kit (TaKaRa, China) was used for reverse transcription-PCR (RT-PCR) or real-time quantitative PCR (qPCR) experiments, respectively.

\section{RACE PCR}

The Es-CLSP cDNA sequence was extended using RACE PCR and a total of 2 genespecific primers (Gp5-1, Gp3-1; Table 1) based upon the original expressed sequence tag (contig612) from the cDNA library was previously constructed (Jiang et al., 2009). The 3'and 5'-RACE reactions were performed using the SMARTer ${ }^{\mathrm{TM}}$ RACE cDNA amplification kit (Clontech, USA) according to manufacturer's protocol. The PCR program was performed as a touchdown PCR, according to manufacturer's protocol. The amplified cDNA fragments were cloned into the PMD18-T vector (TaKaRa), and the recombinants were identified by blue/ white screening and confirmed by PCR. Plasmids containing the inserted Es-CLSP fragment were used as the template for DNA sequencing.

\section{Multiple sequence alignment and phylogenetic analyses}

The full-length multiple alignment of the Es-CLSP sequence was compared with 
that of other species by using the BLAST program (Altschul et al., 1997). Amino acid sequences from various species were retrieved from the NCBI GenBank and analyzed using the ClustalW 2.0 Multiple Alignment program (Li, 2003). A neighbor-joining (NJ) phylogenetic tree was constructed using the MEGA software version 4.0 (Tamura et al., 2007). The reliability of the branching was tested using bootstrap re-sampling (with 1000 pseudo replicates).

\begin{tabular}{llc}
\multicolumn{2}{l}{ Table 1. Sequences of primers. } & \\
\hline Primers & Sequence & Code \\
\hline Gene-specific primers for Es-CLSP cloning & 5'-AGACTTCCAGTGCCACGACTTGCCG-3' & Gp5-1 \\
Gene-specific primer pairs for RACE & 5'-ACATCCGTCTGAACGAAGATACCCA-3' & Gp3-1 \\
RT-PCR and qPCR primers & & \\
Es-CLSP 5'primer & 5'-TGACCTGGGTATCTTCGTTCAGACG-3' & Q-R \\
Es-CLSP 3'primer & 5'-GGTGAAGTCATCCCGCATCGT-3' & Q-F \\
$\beta$-actin for RT-PCR and qRT-PCR & & $\beta-R$ \\
$\beta$-actin R & 5'-GGGGTGTTGAAGGTCTCGGA-3' & $\beta-F$ \\
$\beta$-actin F & 5'-CCTCACCCTCAAATACCCCAT-3' & T7 \\
Primers for sequencing & 5'-TAATACGACTCACTATAGG-3' & SP6 \\
T7 & 5'-ATTTAGGTGACACTATAGAA-3' & \\
SP & & \\
\hline
\end{tabular}

\section{Organ distribution and qPCR analysis}

The organ-dependent Es-CLSP mRNA expression was measured by qPCR by using the following method. Briefly, first-strand cDNA was prepared as described above. Genespecific primers (Q-F and Q-R; Table 1) were designed based upon the cloned Es-CLSP cDNA to produce a 215-bp amplicon. All qPCR were performed in triplicate by using extracted RNA (pooled) of the same concentration. Real-time qPCR was performed in a $1000^{\mathrm{TM}}$ Thermal Cycler (BioRad CFX 96 ${ }^{\mathrm{TM}}$ Real-Time System) according to the manufacturer instructions. The final volume of each reaction was $25 \mu \mathrm{L}$, which contained $12.5 \mu \mathrm{L} 2 \mathrm{X}$ SYBR Premix ExTaq (TaKaRa), $1.0 \mu \mathrm{L}$ diluted cDNA template, $10.5 \mu \mathrm{L}$ PCR-grade water, and $0.5 \mu \mathrm{L} 10$ $\mu \mathrm{M}$ primer dilution. PCR conditions were as follows: $95^{\circ} \mathrm{C}$ for $10 \mathrm{~s}$, followed by 35 cycles of $95^{\circ} \mathrm{C}$ for $5 \mathrm{~s}$, and a $0.5^{\circ} \mathrm{C} / 5$-s incremental increase from $60^{\circ} \mathrm{C}$ to $95^{\circ} \mathrm{C}$ that lasted $30 \mathrm{~s}$ per cycle. The primers $\beta$-actin $\mathrm{F}$ and $\beta$-actin $\mathrm{R}(\beta-\mathrm{F}, \beta-\mathrm{R})$ were designed based on a cloned $E$. sinensis $\beta$-actin cDNA fragment to produce a 276 -bp amplicon. PCR was performed according to the PrimeScript Real-time PCR Kit protocol. Samples were run in triplicate and normalized to the selected control gene $\beta$-actin. Es-CLSP expression levels were calculated by the $2^{-\Delta \Delta \mathrm{Ct}}$ comparative CT method (Schmittgen and Livak, 2008). Mean and standard deviations were calculated from triplicate experiments and presented as the $\mathrm{n}$-fold difference in expression relative to $\beta$-actin. Data was analyzed using the CFX Manager ${ }^{\mathrm{TM}}$ software version 1.0.

\section{Statistical analyses}

Statistical analyses were performed using the SPSS software version 13.0. Data are reported as means \pm 1 standard error (SE). Statistical significances were determined by oneway ANOVA (Tamhane, 1977) and post-hoc Duncan multiple range tests. Significance was set at $\mathrm{P}<0.05$. 


\section{RESULTS}

\section{Cloning and characterization of the Es-CLSP gene}

The results of BLAST revealed that this fragment was highly homologous to the CLSP of Paralithodes camtschaticus (99\%) and chymotrypsin of Scylla paramamosain (98\%). The full length of the cDNA encoding Es-CLSP was 990 bp in length, which began with a methionine codon at position 24 and ends with a TGA termination codon at position 831. This cDNA contained a predicted open reading frame of $813 \mathrm{bp}$, which encodes 270-amino acid-long polypeptides. The GenBank accession number is HM345981.

Using the InterPro search database, typical motifs of signal sequences of SPs were identified in Es-CLSP at the histidine active site (81-86 aa), the serine active site (216-227 aa), and the trypsin domain profile (mature domain, 45-267 aa). Potential phosphorylation sites were identified from Es-CLSP through prosite scan at PBIL (Pôle Bio-Informatique Lyonnais). Es-CLSP contained ATP/GTP-binding site motif A (P-loop) at 17-24 aa (GxxxxGKS), casein kinase II phosphorylation site at 113-116, 118-121, and 201-204 aa (TXXD/TXXE), and N-myristoylation site at 17-22, 47-52, 72-77, 213-218, 223-228, and 239-244 aa (SXR/ SXK). Es-CLSP contained the catalytic triad of the typical SPs (H, D, and S). The deduced amino acid sequence and Es-CLSP motifs are shown in Figure 1.

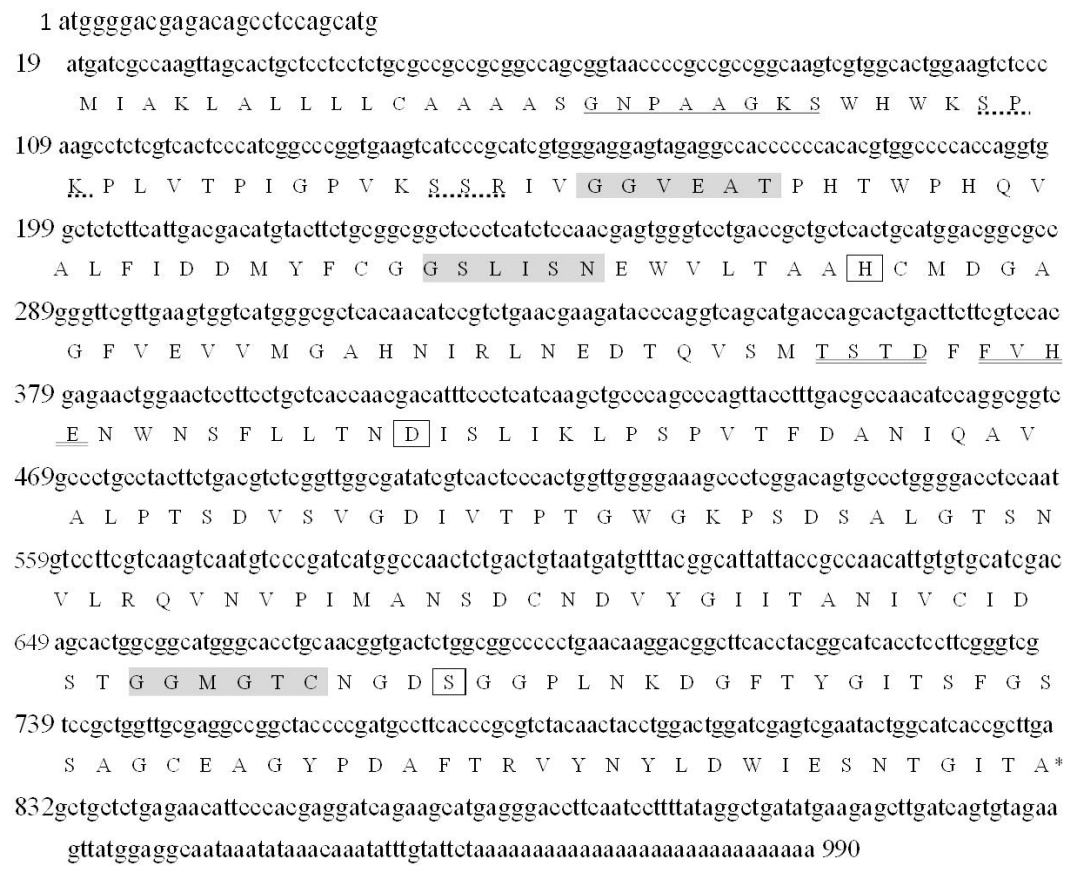

Figure 1. Nucleotide and deduced amino acid sequences of Es-CLSP. The lower case letters indicate 5' and 3' untranslated regions and upper case letters indicate the coding region. The nucleotides are numbered on the left. The putative ATP/GTP-binding site motif A (P-loop, 17-24 aa) is underlined. The N-myristoylation site is black shadowed, the casein kinase II phosphorylation site is double-underlined and the kinase $\mathrm{C}$ phosphorylation site is shown in dotted line. The catalytic triad $(\mathrm{H}, \mathrm{D}$, and $\mathrm{S})$ is in box. 


\section{Similarity and phylogenetic analyses}

Blast analysis revealed that Es-CLSP (GenBank No. AEF33836) shared high similarity with CLSPs from other species (Figure 2), including Uca pugilator (79\%), Scylla paramamosain (76\%), Litopenaeus vannamei (76\%), Fenneropenaeus chinensis (72\%), Salmo (61\%), Gallus gallus (52\%), Mus musculus (52\%), and Homo sapiens (51\%). Furthermore, Es-CLSP shared $>40 \%$ identity with CLSP from certain other species. The phylogeny of CLSP family is shown in Figure 3. The NJ tree could be divided into 2 groups: invertebrate CLSP and vertebrate CLSP. Invertebrate CLSP consists of crabs, shrimps, and other insects. Subgroup 2 consists of fishes, animals, and humans. Es-CLSP shows higher homology to CLSP of $U$. pugilator; hence, it may be a new member of CLSP protease.

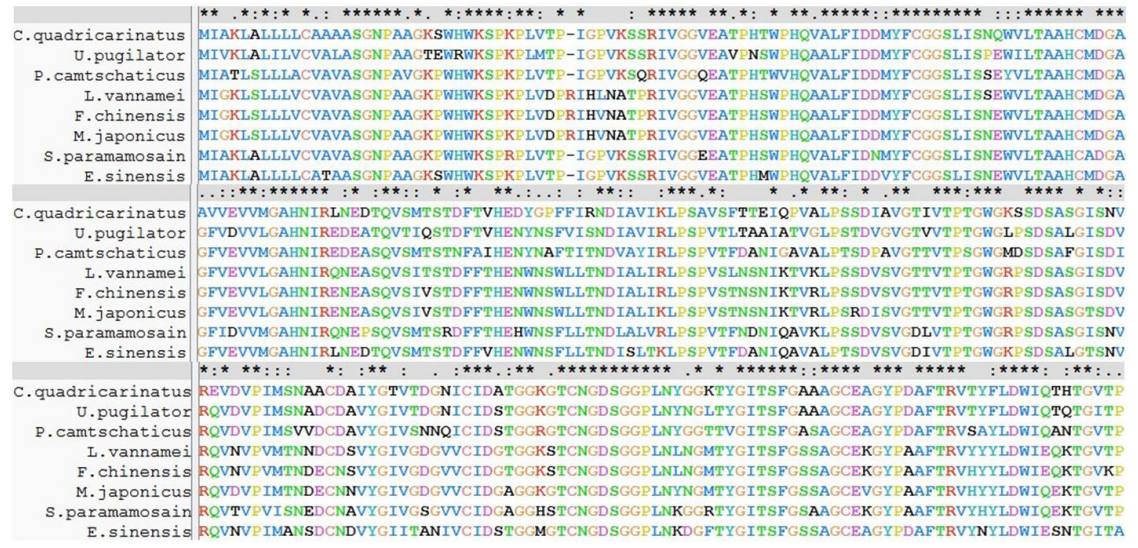

Figure 2. Multiple alignment of crustacean CLSP amino acid sequences. Identical (*) and similar (. or :) amino acid residues are indicated. GenBank accession numbers can be seen in Figure 3. The catalytic triad (H, D, and S) is shown with an arrow, and the substrate-binding pocket residues are shown with a black triangle.
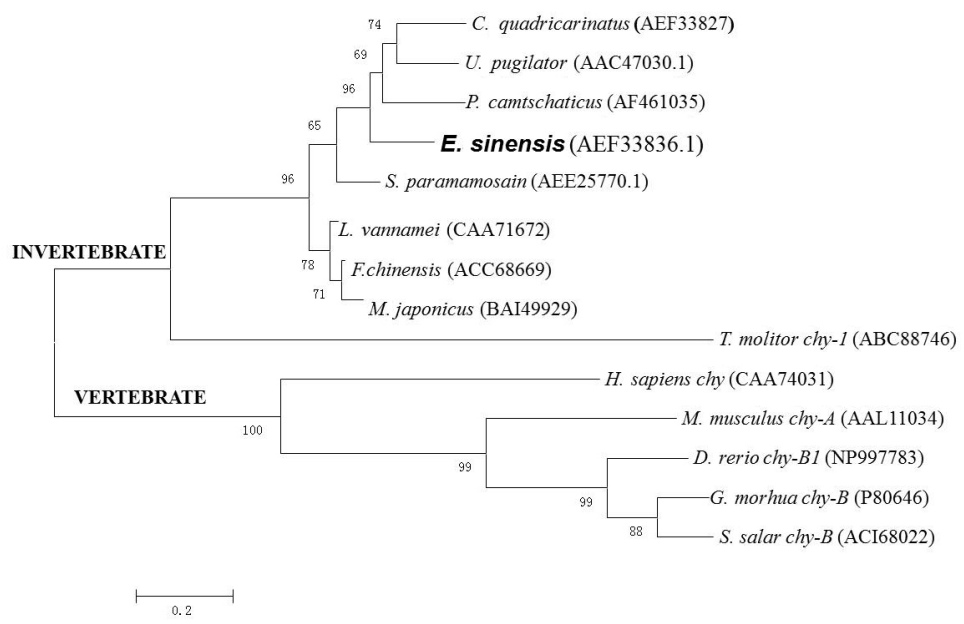

Figure 3. Neighbor-joining phylogenetic tree of the Es-CLSP protein and similar proteins. The phylogram topology was representative for 1000 bootstrap replicates. The GenBank accession number is shown in brackets after each species. 


\section{Organ-specific expression of Es-CLSP}

To determine the expression profiles of Es-CLSP in E. sinensis, total RNA was extracted from the normal Chinese mitten crab. As shown in Figure 4, CLSP was distributive in all tissues, including the hepatopancreas, hemolymph, heart, gills, stomach, intestines, testes, and ovaries. The expression level in the hemolymph and hepatopancreas were considerably higher than that in other tissues.

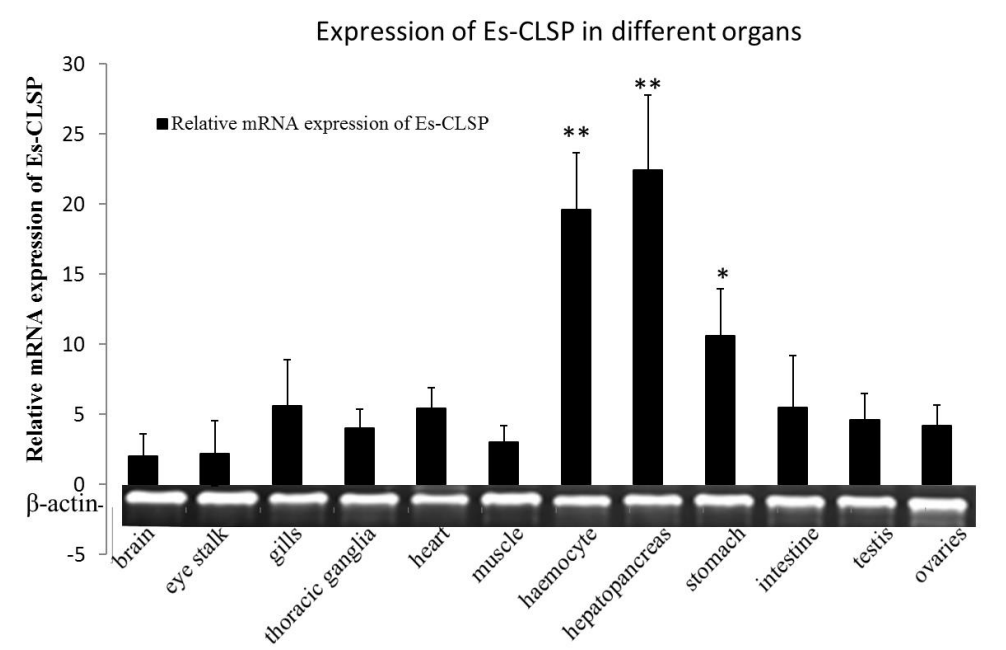

Figure 4. Expression of Es-CLSP in different organs. *Significant difference $(\mathrm{P} \leq 0.05)$. ${ }^{*}$ Highly significant difference $(\mathrm{P} \leq 0.01)$.

\section{Temporal expression profiles after $\boldsymbol{V}$. anguillarum challenge}

Es-CLSP temporal expression level, as measured by qPCR following $V$. anguillarum challenge (Figure 5). Interestingly, Es-CLSP temporal expression was significantly upregulated as soon as $2 \mathrm{~h}$ post $V$. anguillarum injection, and its expression was significantly greater than the vehicle control after $2,4,8,12,24$, and $48 \mathrm{~h}$ post $V$. anguillarum stimulation $(\mathrm{P}<$ 0.05 ); this peaked to 4.8 -fold of that of the vehicle control after $8 \mathrm{~h}$ post-injection.

\section{DISCUSSION}

The CLSP family participates in a wide range of biological reactions and is found in both prokaryotic and eukaryotic organisms (Hedstrom, 2001; Krem and Di Cera, 2001). In this regard, the full-length cDNA sequence of CLSP was isolated from the hepatopancreas of E. sinensis (Figure 1). The deduced amino acid sequence of Es-CLSP showed several structural features that are typical of SPs (Figures 1 and 2). Although the substrate-binding pocket residues of SPs are variable, in CLSP, they usually consist of Ser, Gly, and Ala/Gly (Colebatch et al., 2002). One of the substrate-binding pocket residues of Es-CLSP was replaced by a Gly residue (at position 216); this substitution is also found in the trypsin and CLSP in the gut of Mayetiola destructor (Zhu et al., 2005), a small amino acid found in vertebrate CLSP. It also 
appears in Es-CLSP, and therefore, we classified this gene as a new CLSP.

BlastP results showed Es-CLSP was highly homologous to CLSP from other kind of shrimps. Similarity analysis revealed that Es-CLSP has several typical structural features of SPs (Kraut, 1977; Perona and Craik, 1995), such as the catalytic triad (H, D, and S), conserved serine catalytic site, and 6 cysteine residues. In the conserved trypsin-like SP domain, there are 6 cysteine residues common to all invertebrate CLSPs, and these are believed to form 3 disulfide bonds (Colebatch et al., 2002). In addition to the 3 pairs of conserved cysteine residues, there is another free cysteine residue in the signal peptide. Cysteine residues exist in many insect SPs; however, the exact role and pairing mode of these cysteine residues remain unclear.

Phylogenetic analysis revealed that Es-CLSP clustered with CLSP from U. pugilator, P. camtschaticus, F. chinensis, Marsupenaeus japonicas, Cherax quadricarinatus, and L. vannamei. Hence, it can be said that Es-CLSP belongs to the SP subfamily. The Es-CLSP clustered with invertebrate CLSP, whereas another branch is the vertebrate CLSP, which supports the traditional evolutionary theory (Figure 3).

The results of tissue expression analysis using RT-PCR showed that Es-CLSP mRNA was detected in all tissues, including the hepatopancreas, stomach, and gonads. CLSP from other crustaceans were also detected in the hepatopancreas (Zou et al., 2006; Kalinina et al., 2008; Shi et al., 2008; Danwattananusorn et al., 2009). Western blot analysis of extracts from F. chinensis showed that CLSP was mainly distributed in the hepatopancreas, stomach, and intestine (Smith et al., 2003; Shi et al., 2008). Our results on transcriptional expression also suggested that Es-CLSP was synthesized in the hepatopancreas, and then secreted and transported to the stomach, intestine, and other tissues. Expression analysis using RT-PCR and qPCR revealed that Es-CLSP is expressed in defense-related tissues, including the hemocytes and hepatopancreas (Figure 4).

The mRNA expression of Es-CLSP in the hepatopancreas was upregulated after $V$. anguillarum challenge. The qPCR results also showed that the expression level did not have significant change after the same volume of saline injection. Hence, the upregulation was the result of the $V$. anguillarum injection and not the injury. Time-elapsed expression analysis showed that after $V$. anguillarum injection, the Es-CLSP mRNA expression level in the injection group displays a time-related and dose-dependent expression pattern (Figure 5). V. anguillarum injection, following mRNA transcription in response to pathogen infection, ultimately led to a gradual increase in the mRNA levels to the normal level or even to a higher level. These results were consistent with the mRNA expression profiles in other species. Further SPs can be divided into 2 types of molecules according to their domains: single-trypsin domain SPs (single-domain SPs) and clip-trypsin domain SPs (clip-domain SPs) (Piao et al., 2005; Volz et al., 2005). In arthropods, clip-domain SPs have been proved to function in mediating innate immunity and embryonic development (Gorman and Paskewitz, 2001; Piao et al., 2005; Volz et al., 2005; Zou et al., 2006; Ren et al., 2009). Hence, taking into consideration the EsCLSP domain structure and the challenge profiles, it is clear that Es-CLSP plays an important role in the crab immune response by participating in physiological processes, including hemolymph coagulation, melanization of pathogen surfaces, and antimicrobial peptide synthesis.

In conclusion, a CLSP was identified in the Chinese crab E. sinensis. The entire length of this SP was cloned, and its expression pattern in response to $V$. anguillarum challenge was described for the first time. Further study concerning its mechanism of action in the innate immune system may elucidate its exact immunological and offer a potential vehicle for use in the prevention of $V$. anguillarum infections in aquaculture stocks. 


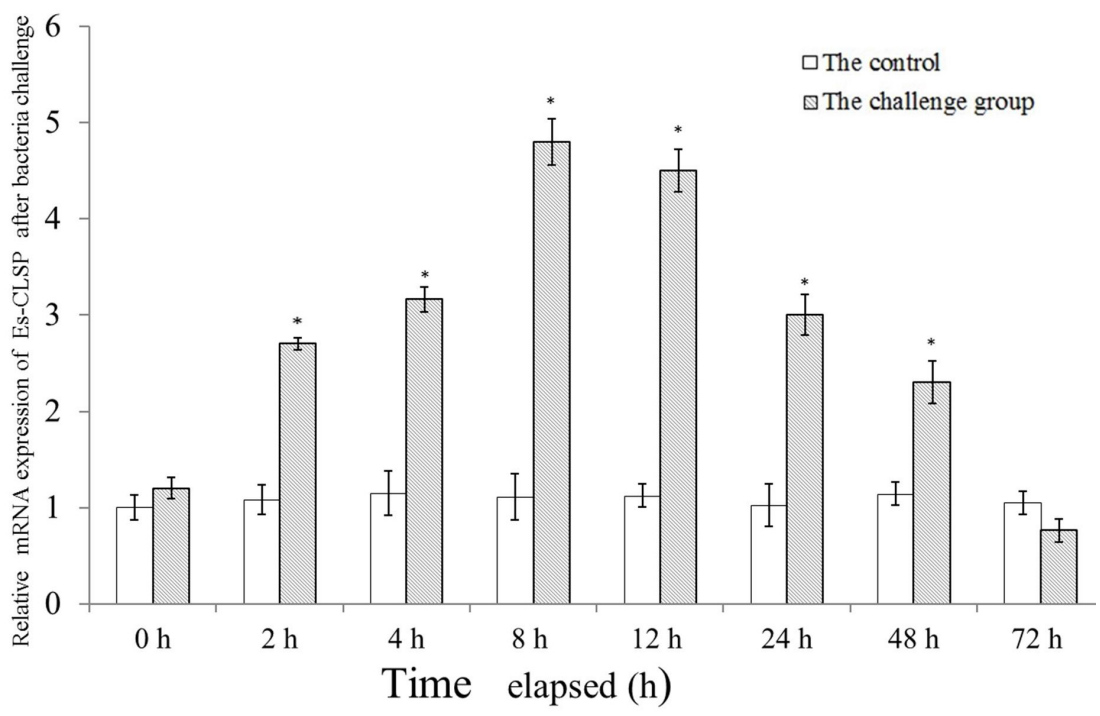

Figure 5. Temporal expression profile of Es-CLSP after Vibrio anguillarum challenge. *Significant difference (P $<0.05)$

\section{ACKNOWLEDGMENTS} (\#505001).

Research supported by grants from the doctoral fund of Xinxiang Medical University

\section{REFERENCES}

Altschul SF, Madden TL, Schaffer AA, Zhang J, et al. (1997). Gapped BLAST and PSI-BLAST: a new generation of protein database search programs. Nucleic Acids Res. 25: 3389-3402.

Barrett AJ and Rawlings ND (1995). Families and clans of serine peptidases. Arch. Biochem. Biophys. 318: 247-250.

Botos I, Meyer E, Nguyen M, Swanson SM, et al. (2000). The structure of an insect chymotrypsin. J. Mol. Biol. 298: 895-901.

Broehan G, Zimoch L, Wessels A, Ertas B, et al. (2007). A chymotrypsin-like serine protease interacts with the chitin synthase from the midgut of the tobacco hornworm. J. Exp. Biol. 210: 3636-3643.

Chen DW, Zhang M and Shrestha S (2007). Compositional characteristics and nutritional quality of Chinese mitten crab (Eriocheir sinensis). Food Chem. 103: 1343-1349.

Colebatch G, Cooper P and East P (2002). cDNA cloning of a salivary chymotrypsin-like protease and the identification of six additional cDNAs encoding putative digestive proteases from the green mirid, Creontiades dilutus (Hemiptera: Miridae). Insect Biochem. Mol. Biol. 32: 1065-1075.

Danwattananusorn T, Kondo H, Aoki T and Hirono I (2009). Molecular cloning, characterization and expression analysis of a chymotrypsin-like serine protease from kuruma shrimp Marsupenaeus japonicus. Fish. Sci. 75: 1231-1238.

de Morais GS, Vitorino R, Domingues R, Tomer K, et al. (2005). Proteomics of immune-challenged Drosophila melanogaster larvae hemolymph. Biochem. Biophys. Res. Commun. 328: 106-115.

Finnerty CM, Karplus PA and Granados RR (1999). The insect immune protein scolexin is a novel serine proteinase homolog. Protein Sci. 8: 242-248.

García-Ulloa GM, López-Chavarín HM, Rodríguez-González H and Villarreal-Colmenares H (2003). Growth of redclaw crayfish Cherax quadricarinatus (Von Martens 1868) (Decapoda: Parastacidae) juveniles fed isoproteic diets with partial or total substitution of fish meal by soya bean meal: preliminary study. Aquacult. Nutr. 9: 25-31. 
Gorman MJ and Paskewitz SM (2001). Serine proteases as mediators of mosquito immune responses. Insect Biochem. Mol. Biol. 31: 257-262.

Hedstrom L (2001). An Overview of Serine Proteases, Current Protocols in Protein Science. John Wiley \& Sons, Inc., New York.

Herrero S, Combes E, Van Oers MM, Vlak JM, et al. (2005). Identification and recombinant expression of a novel chymotrypsin from Spodoptera exigua. Insect Biochem. Mol. Biol. 35: 1073-1082.

Jiang H, Cai YM, Chen LQ, Zhang XW, et al. (2009). Functional annotation and analysis of expressed sequence tags from the hepatopancreas of mitten crab (Eriocheir sinensis). Mar. Biotechnol. 11: 317-326.

Kalinina MV, Vinnikova NA and Semen'kova EG (2008). Gonadogenesis and color characteristics of ovaries in Japanese mitten crab Eriocheir japonicus. Ontogenez 39: 58-65.

Kraut J (1977). Serine proteases: structure and mechanism of catalysis. Annu. Rev. Biochem. 46: 331-358.

Krem MM and Di Cera E (2001). Molecular markers of serine protease evolution. EMBO J. 20: 3036-3045.

Li KB (2003). ClustalW-MPI: ClustalW analysis using distributed and parallel computing. Bioinformatics 19: 1585-1586.

Miranda R, Zamudio FZ and Bravo A (2001). Processing of Cry $1 \mathrm{Ab} \delta$-endotoxin from Bacillus thuringiensis by Manduca sexta and Spodoptera frugiperda midgut proteases: role in protoxin activation and toxin inactivation. Insect Biochem. Mol. Biol. 31: 1155-1163.

Moffitt KL, Walker B and Martin SL (2012). Chymotrypsin-like serine proteinases are involved in the maintenance of cell viability. Biochimie 94: 2582-2589.

O'Connell AR, Lee BW and Stenson-Cox C (2006). Caspase-dependant activation of chymotrypsin-like proteases mediates nuclear events during Jurkat T cell apoptosis. Biochem. Biophys. Res. Commun. 345: 608-616.

Perona JJ and Craik CS (1995). Structural basis of substrate specificity in the serine proteases. Protein Sci. 4: 337-360.

Piao S, Kim D, Won PJ, Leul LB, et al. (2005). Overexpression and preliminary X-ray crystallographic analysis of prophenoloxidase activating factor II, a clip domain family of serine proteases. Biochim. Biophys. Acta 1752: 103-106.

Ren Q, Xu ZL, Wang XW, Zhao XF, et al. (2009). Clip domain serine protease and its homolog respond to Vibrio challenge in Chinese white shrimp, Fenneropenaeus chinensis. Fish Shellfish. Immunol. 26: 787-798.

Sánchez-Paz A (2010). White spot syndrome virus: an overview on an emergent concern. Vet. Res. 41: 43.

Schmittgen TD and Livak KJ (2008). Analyzing real-time PCR data by the comparative C(T) method. Nat. Protoc. 3: 1101-1108.

Sellos D and Van Wormhoudt A (1992). Molecular cloning of a cDNA that encodes a serine protease with chymotryptic and collagenolytic activities in the hepatopancreas of the shrimp Penaeus vanameii (Crustacea, Decapoda). FEBS Lett. 309: 219-224.

Sellos D and Van Wormhoudt A (1999). Polymorphism and evolution of collagenolytic serine protease genes in crustaceans. Biochim. Biophys. Acta 1432: 419-424.

Shi XZ, Zhao XF and Wang JX (2008). Molecular cloning and expression analysis of chymotrypsin-like serine protease from the Chinese shrimp, Fenneropenaeus chinensis. Fish Shellfish. Immunol. 25: 589-597.

Smith VJ, Brown JH and Hauton C (2003). Immunostimulation in crustaceans: does it really protect against infection? Fish Shellfish. Immunol. 15: 71-90.

Söderhäll K and Cerenius L (1998). Role of the prophenoloxidase-activating system in invertebrate immunity. Curr. Opin. Immunol. 10: 23-28.

Sveinsdóttir H, Thorarensen H and Gudmundsdóttir Á (2006). Involvement of trypsin and chymotrypsin activities in Atlantic cod (Gadus morhua) embryogenesis. Aquaculture 260: 307-314.

Tamhane AC (1977). Multiple comparisons in model i one-way anova with unequal variances. Commun. Stat. - Theor. Methods 6: 15-32.

Tamura K, Dudley J, Nei M and Kumar S (2007). MEGA4: Molecular Evolutionary Genetics Analysis (MEGA) software version 4.0. Mol. Biol. Evol. 24: 1596-1599.

Van Wormhoudt A, Le CP and Sellos D (1992). Purification, biochemical characterization and N-terminal sequence of a serine-protease with chymotrypsic and collagenolytic activities in a tropical shrimp, Penaeus vannamei (Crustacea, Decapoda). Comp. Biochem. Physiol. B 103: 675-680.

Volz J, Osta MA, Kafatos FC and Muller HM (2005). The roles of two clip domain serine proteases in innate immune responses of the malaria vector Anopheles gambiae. J. Biol. Chem. 280: 40161-40168.

Yadav RP, Patel AK and Jagannadham MV (2011). Purification and biochemical characterization of a chymotrypsin-like serine protease from Euphorbia neriifolia Linn. Process Biochem. 46: 1654-1662.

Zhu YC, Liu X, Maddur AA, Oppert B, et al. (2005). Cloning and characterization of chymotrypsin- and trypsin-like cDNAs from the gut of the Hessian fly [Mayetiola destructor (Say)]. Insect Biochem. Mol. Biol. 35: 23-32.

Zou Z, Lopez DL, Kanost MR, Evans JD, et al. (2006). Comparative analysis of serine protease-related genes in the honey bee genome: possible involvement in embryonic development and innate immunity. Insect Mol. Biol. 15: 603-614.

Genetics and Molecular Research 13 (2): 3885-3894 (2014) 